\section{(O) OPEN ACCESS}

\title{
A thromboembolic model for the efficacy and safety evaluation of combined mechanical and pharmacologic revascularization strategies
}

\author{
Matthew J Gounis, ${ }^{1}$ Raul G Nogueira, ${ }^{2}$ Manik Mehra, ${ }^{1}$ Juyu Chueh, ${ }^{1}$ Ajay K Wakhloo ${ }^{1}$
}

${ }^{1}$ Department of Radiology, New England Center for Stroke Research, University of Massachusetts Medical School, Worcester, Massachusetts, USA ${ }^{2}$ Marcus Stroke \& Neuroscience Center, Grady Memorial Hospital, Department of Neurology, Neurosurgery, and Radiology, Emory University, Atlanta, Georgia, USA

\section{Correspondence to}

Dr M J Gounis, Department of Radiology, New England Center for Stroke Research, University of Massachusetts, 55 Lake Avenue North, SA-107R Worcester, MA 01655, USA: matt.gounis@umassmed.edu

Received 29 May 2012 Revised 8 August 2012 Accepted 9 August 2012 Published Online First 8 September 2012

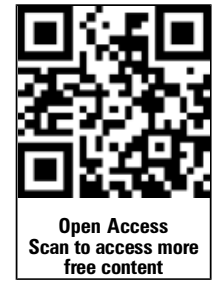

\section{ABSTRACT}

Background and purpose Recanalization strategies mediated by intra-arterial fibrinolytic therapy in combination with mechanical clot disruption may be a more effective treatment approach than either therapy used alone. There are few preclinical animal models to evaluate these strategies. Here we report on a model to simultaneously evaluate both of these treatment approaches.

Methods Allogeneic clot was injected through the $6 \mathrm{~F}$ guide catheter after creating $>50 \%$ luminal stenosis of the common carotid arteries of New Zealand White rabbits. The stenosis was released after $1 \mathrm{~h}$, allowing sufficient time for clot-vessel wall interaction. Occlusion was confirmed and each vessel was assigned to receive either balloon angioplasty alone, intra-arterial tissue plasminogen activator (tPA, Alteplase, Genentech, San Francisco, California, USA), tPA delivery through prototype balloon infusion wire (NIT Therapeutics, Pittsburgh, Pennsylvania, USA), partial stent deployment or partial stent deployment with locally delivered tPA. The negative control received no treatment.

Results In vivo revascularization Thrombolysis in Cerebral Infarction (TICI) score revealed that the balloon infusion wire achieved a stable and higher revascularization score of $\mathrm{TICI} 2 \mathrm{~B}$, with a lower dose of tPA in comparison with other treatment strategies. All treatment strategies resulted in endothelial denudation and exposure of the internal elastic lamina.

Conclusions The proposed animal model permits reliable and consistent thromboembolic occlusion of the target vasculature and allows for an assessment of both pharmacologic and mechanical revascularization strategies for acute ischemic stroke.

\section{INTRODUCTION}

Current treatment strategies employed for acute ischemic stroke remain pharmacologic thrombolysis and mechanical thrombectomy. Intravenous thrombolytic tissue plasminogen activator (tPA, Alteplase, Genentech, San Francisco, California, USA) remains the only Food and Drug Administration approved therapy for acute ischemic stroke for the past 16 years, ${ }^{1}$ nevertheless its use remains limited to only approximately $5 \%$ of patients presenting with acute ischemic stroke in the USA. ${ }^{2}{ }^{3}$ Intra-arterial thrombolytic administration allows for localized delivery of a reduced drug dose at a much higher concentration that may help in more effective lysis of the clot burden. ${ }^{4}$ Multimodal treatment strategies involving more aggressive endovascular mechanical clot disruption such as angioplasty or stenting when used in conjunction with even lower doses of intra-arterial thrombolytics may help achieve earlier recanalization and reduce the risk of hemorrhage that is inherent with the use of larger doses of systemic and local thrombolytic therapy alone. ${ }^{5-7}$

To test this hypothesis in vivo, we require a suitable animal model for evaluation of mechanical clot disruption coupled with thrombolytic therapy. The prerequisites of such a model involve: (1) target vessel having a diameter similar to the human middle cerebral artery; (2) methodology that permits consistent vascular occlusion at a prespecified location; (3) ability to access the lesion endovascularly; and (4) ability of recombinant tPA to have a comparable fibrinolytic effect in the animal species as seen in humans. We selected the rabbit common carotid artery (CCA) for this research as the mean dimensions of the rabbit CCA are $2.5 \mathrm{~mm}$ and comparable with the human middle cerebral artery. Rabbits are amendable to $6 \mathrm{~F}$ transfemoral catheter access and the efficacy of tPA in this species is well studied. ${ }^{8}$ In this model, we evaluated various combinations of mechanical and pharmacologic revascularization strategies.

\section{MATERIALS AND METHODS}

All procedures were performed in accordance with the protocol approved by our university's Institutional Animal Care and Use Committee. Eleven New Zealand white rabbits (male, mean weight $3.53 \mathrm{~kg}$ ) were used for pharmacomechanical recanalization in 22 vessels.

\section{Clot preparation}

Silicone tubing with an inner diameter of $3.2 \mathrm{~mm}$ was rinsed with $70 \%$ alcohol followed by $0.9 \%$ saline prior to use. Whole blood was collected from an anaesthetized donor rabbit, and mixed with the anticoagulant citrate dextrose solution in a 10:1 ratio. The blood/anticoagulant citrate dextrose mixture and thrombin $(2.5 \mathrm{NIH} \mathrm{U} / \mathrm{ml}$ blood) were injected into the silicone tubing simultaneously to rapidly initiate clotting. The clotted material was allowed to mature for $1 \mathrm{~h}$ prior to use. During aging, shrinkage of the clot resulted in diameter reduction from $3.2 \mathrm{~mm}$ to $2.7 \mathrm{~mm}$. The clot was cut to have a length of $10 \mathrm{~mm}$ for creation of the vascular occlusion. 


\section{Animal procedure}

Anesthesia was induced by an intramuscular injection of ketamine $(35 \mathrm{mg} / \mathrm{kg})$, xylazine $(5 \mathrm{mg} / \mathrm{kg})$ and glycopyrrolate $(0.01 \mathrm{mg} / \mathrm{kg})$, and maintained with mechanical ventilation of oxygen with $1-2 \%$ isoflurane. Continuous monitoring of heart rate, respiration, oxygen saturation level (pulse oximetry), end tidal $\mathrm{CO}_{2}$ and temperature allowed real time assessment of the physiologic status of the animal.

A $6 \mathrm{~F}$ haemostatic introducer was placed into the right femoral artery using a modified Seldinger technique following a cut-down. The introducer was secured in place with the distal aspect of the femoral artery ligated. A 6 F catheter was advanced over the guidewire under x-ray fluoroscopy (Phillips FD20, Best, The Netherlands). Heparin (100 IU/kg) was delivered intravenously. The intervention was done under angiographic guidance.

Both CCAs were exposed through a ventral midline neck incision and a 3-0 silk suture was placed bilaterally to create $>50 \%$ luminal stenosis $2-3 \mathrm{~cm}$ distal to the arterial origin. A clot fragment $(2.7 \mathrm{~mm}$ diameter and $10 \mathrm{~mm}$ length) was injected into each CCA. The clot was delivered with a custom syringe having an enlarged outlet to prevent clot fragmentation and the syringe was connected directly to the catheter. Prior to initiating the study, the clot was injected through the catheter into a Petri dish to ensure that the clot remained intact and preserved its original dimensions. Using this method, we found the maximum diameter of the elastic clot that could be delivered through the catheter without fragmentation was $2.7 \mathrm{~mm}$. After injecting the clot into the CCA, diagnostic angiography was performed to confirm the occlusion. Approximately $1 \mathrm{~h}$ post occlusion, allowing sufficient time for vessel lumen-clot interaction, the external sutures inducing the luminal stenosis were released (figure 1). The treatment strategies were evaluated as described in the section below.

The ability of the treatment modality to revascularize the vessel was evaluated at the end of the procedure by assigning a Thrombolysis in Cerebral Infarction (TICI) score based on the final vessel angiogram. ${ }^{9}$

\section{Revascularization strategies evaluated}

Treatment strategies evaluated were either purely mechanical, purely pharmacologic thrombolysis or a combination of the two treatment strategies.

Purely mechanical treatment was evaluated using a compliant Hyperglide $4 \times 20 \mathrm{~mm}$ balloon (eV3 Neurovascular Inc, Irvine, California, USA, $n=3$ ). Purely pharmacologic thrombolysis $(n=4)$ was evaluated by selective intra-arterial tPA delivery at the occlusion site.

A novel treatment evaluated was the balloon infusion wire (NeuroInterventional Therapeutics Inc, Pittsburgh, Pennsylvania, USA, $n=3$ ), an investigational device which consists of a wire body and a distal balloon. The balloon is inflated by injecting the diagnostic or therapeutic fluid agent which then weeps through the balloon orifices and thus can be used to evaluate combined mechanical and pharmacologic tPA medicated thrombolytic approaches.

Partial stent deployment entailed the temporary unconstraining of the self-expanding closed cell design stent (Enterprize VRD; $4.5 \mathrm{~mm} \times 37 \mathrm{~mm}$ Codman and Shurtleff, Raynham, Massachusetts, USA, $n=5$ ) within the clot to create a temporary bypass for $5 \mathrm{~min}$ to test the hypothesis that temporary flow restoration and clot maceration against the vessel wall may result in autolysis.

A further evaluation of the effect of partial stent deployment coupled with site directed intra-arterial tPA was also made $(n=2)$ to evaluate whether mechanically exposing a larger surface area of the clot to tPA results in a more expedited revascularization. The control group $(n=4)$ consisted of the vessel with a confirmed occlusion but received no treatment.

\section{Animal sacrifice and tissue harvest}

At the end of the procedure the animals were injected with $500 \mathrm{IU} / \mathrm{kg}$ of heparin. Following euthanasia by injecting sodium pentobarbital $(150 \mathrm{mg} / \mathrm{kg})$, normal saline was perfused through the ascending aorta via a catheter until the effluent from the external jugular vein ran clear. This was followed by perfusion fixation using $4 \%$ paraformaldehyde. Both CCAs were carefully harvested. The segment of the vessel that received treatment was identified by carefully matching with the angiography.

\section{Histological analysis}

The segment was further divided into a proximal segment which was assigned for histology assessment and processed to obtain 5-7 $\mu \mathrm{m}$ paraffin sections and stained with hematoxylineosin. The sections were imaged at $10 \times$ and $20 \times$ magnification with an Olympus AX-70 microscope (Olympus America, Center Valley, Pennsylvania, USA).

\section{Scanning electron microscopy}

The distal segment was immersion fixed overnight in $2.5 \%$ glutaraldehyde, washed in phosphate buffered saline and

Figure 1 Rabbit thromboembolic occlusion model. (A) Angiogram depicting the bilateral stenosis of the common carotid artery prior to clot injection (arrows). (B) Digital subtraction angiography (DSA) post clot injection (arrow) reveals an occlusion. After allowing $45 \mathrm{~min}$ for vessel-clot interaction, the external sutures were released. (C) Post suture removal DSA reveals a stable occlusion and no distal clot migration.

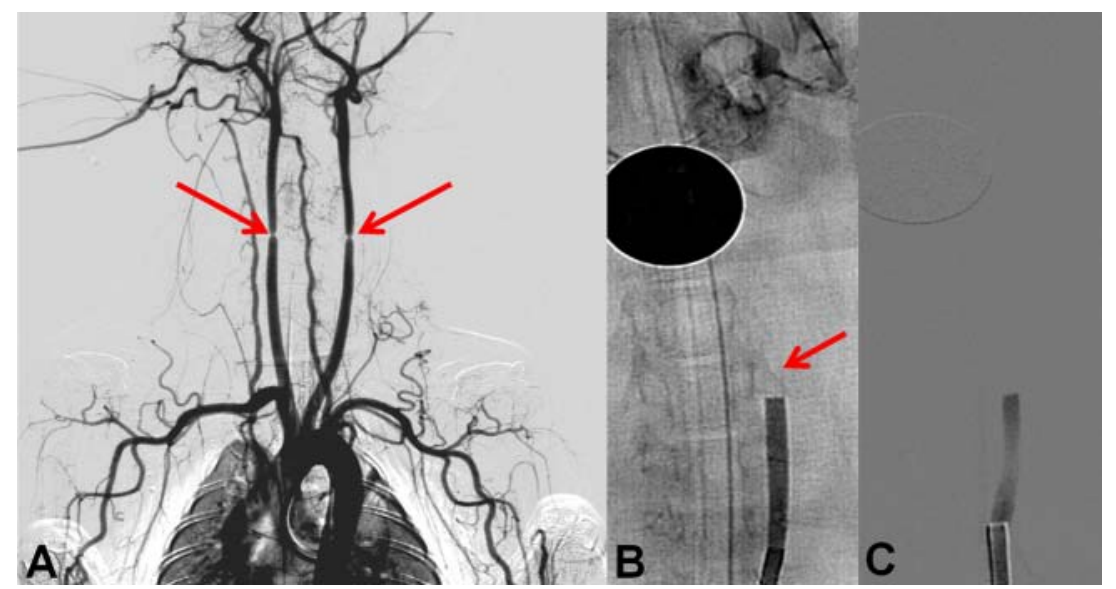


dehydrated through a graded series of ethanol concentrations to $100 \%$ and then critical point dried in carbon dioxide. The arterial samples were mounted on to aluminum stubs, cut longitudinally to expose the luminal surface, coated with silver conductive paste, sputter coated with gold/palladium and examined with an autoscan scanning electron microscopy (SEM) (ETEC Corp, Hayward, California, USA) at $10 \mathrm{KV}$ accelerating voltage. Serial SEM images were acquired at a magnification of $500 \times, 2500 \times$ and $5000 \times$ for each half of the longitudinal segment.

\section{RESULTS}

\section{Evaluation of treatment efficacy}

We successfully created thromboembolic occlusion with TICI 0 flow at the predetermined location within the target vessel. Distal migration of the clot prior to treatment was observed in one case $(4.5 \%)$. In another case, there was spontaneous revascularization of the control vessel, likely due to the systemic effect of $4 \mathrm{mg}$ tPA delivered to the contralateral artery.

Higher doses of intra-arterial tPA $(4 \mathrm{mg})$ were required when thrombolysis was used alone to achieve recanalization in our animal model. The balloon infusion wire resulted in the most consistent and a higher grade of recanalization (TICI 2B) with $2.75 \pm 1.08 \mathrm{mg}$ tPA in comparison with balloon angioplasty, partial stent deployment with and without tPA, or intra-arterial tPA alone. The final revascularization score is presented in table 1 and a summary bar graph representation in figure 2. Representative results of the device efficacy and safety evaluations are presented in figure 3 .

\section{Safety of the treatment strategy}

Evaluation of the histology and luminal SEM data revealed that the endovascular damage caused by all treatment modalities was similar, with endothelial cell denudation and exposure of the internal elastic lamina. The damage was confined to the operating area of the device; therefore, in the case of catheter directed tPA, the vessel insult was confined to a small area. Also, in a single case, angioplasty alone resulted in a focal breach of the internal elastic lamina. A phenomenon described as medial acellularity was observed sporadically with balloon angioplasty (figure 4) and during operation of the balloon infusion wire. ${ }^{10}$ It is described as medial smooth muscle cell death due to balloon mediated arterial hyperdistension. This phenomenon is due to maximal transmission of the tensile stress by balloon inflation to the smooth muscle cells which are firmly attached to the matrix and may result in membrane disruption and cell death.

Table 1 Treatment performed and the final TICl revascularization scores achieved for each arm of the study

\begin{tabular}{lllll}
\hline Group & Treatment & N & $\begin{array}{l}\text { tPA dose (mg) } \\
\text { (mean (SD)) }\end{array}$ & $\begin{array}{l}\text { TICI score (median } \\
\text { (range)) }\end{array}$ \\
\hline 1 & IA tPA only & 4 & $3(1.2)$ & $\mathrm{I}(0-2 \mathrm{~A})$ \\
2 & Angioplasty only & 3 & $\mathrm{~N} / \mathrm{A}$ & $\mathrm{I}(0-2 \mathrm{~B})$ \\
3 & Balloon infusion wire & 3 & $2.75(1.1)$ & 2B (all cases 2B) \\
4 & Partial stent deployment & 5 & $\mathrm{~N} / \mathrm{A}$ & $0(0-2 \mathrm{~A})$ \\
5 & Partial stent deployment & 2 & $3(1.4)$ & $2 \mathrm{~A}(0-2 \mathrm{~A})$ \\
& and IA tPA & 4 & $\mathrm{~N} / \mathrm{A}$ & $0(0-2 \mathrm{~B})$ \\
\hline
\end{tabular}

IA, intra-arterial; N/A, not applicable; TICl, Thrombolysis in Cerebral Infarction; tPA, tissue plasminogen activator.

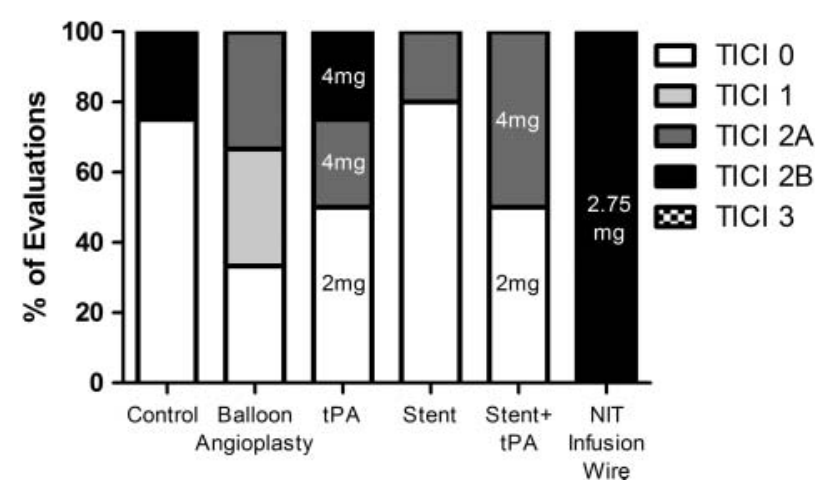

Figure 2 Bar graph representation of treatment efficacy with final Thrombolysis in Cerebral Infarction (TICI) scores post treatment. The dose of tissue plasminogen activator (tPA) administered intra-arterially is noted in the graph bars.

\section{DISCUSSION}

A model system to simultaneously evaluate pharmacologic and mechanical strategies has not been described previously in the literature. Porcine extracranial circulation has been used extensively to evaluate mechanical thrombectomy devices. ${ }^{11}$ However, the 10-fold resistance of porcine plasminogen to activation by recombinant tPA makes them unsuitable for the evaluation of pharmacologic thrombolysis. ${ }^{12}{ }^{13}$ Also, the porcine vasculature is overly susceptible to vasospasm. With the exception of the primates, rabbit fibrinolytic response is closest to that of humans among animal species amenable to endovascular approaches. ${ }^{14}$ The canine model has a weaker fibrinolytic response to tPA and is also a much more expensive model for these evaluations. In our proposed model, the surgical procedure involved is technically less challenging and the vessels are easily accessible for explant and vascular safety analysis. Another advantage of this model is that the number of animals used per study can be limited, since two device evaluations can be carried out per animal, one in each CCA, as long as the purely mechanical device or control evaluation precedes the thrombolytic evaluation, so as not to confound the results with systemic thrombolytic effect.

Also crucial for evaluation of thrombectomy devices are clinically relevant emboli. Many of the previous models evaluating thrombectomy devices have utilized clots prepared with barium sulfate which are radiopaque. This helps in easy visualization of the leading clot edge and is also useful to assess clot engagement, retrieval and fragmentation. ${ }^{11}$ However, this clot model is not ideal for thrombolysis studies. Also, studies characterizing the mechanical properties of the clot have revealed that the addition of barium sulfate results in reduction of clot elasticity. ${ }^{15}$ Finally, the maximal firmness of the rabbit clots with or without thrombin stimulation was found to be similar to that of the human clots. ${ }^{16}$

Evaluation of the histomorphometric and luminal SEM data reveals that the endovascular damage caused by the operation of the balloon infusion wire, balloon angioplasty and partial stent deployment was comparable with the damage caused by conventional endovascular catheter and wire manipulationthat is, endothelial cell denudation and exposure of the internal elastic lamina - an acceptable level of vessel response. ${ }^{17-19}$

The strategy of using reconstrainable self-expanding stents alone as temporary endovascular bypass, impaling the clot circumferentially between the stent interstices and the vessel wall, immediately re-established a channel of flow within the 


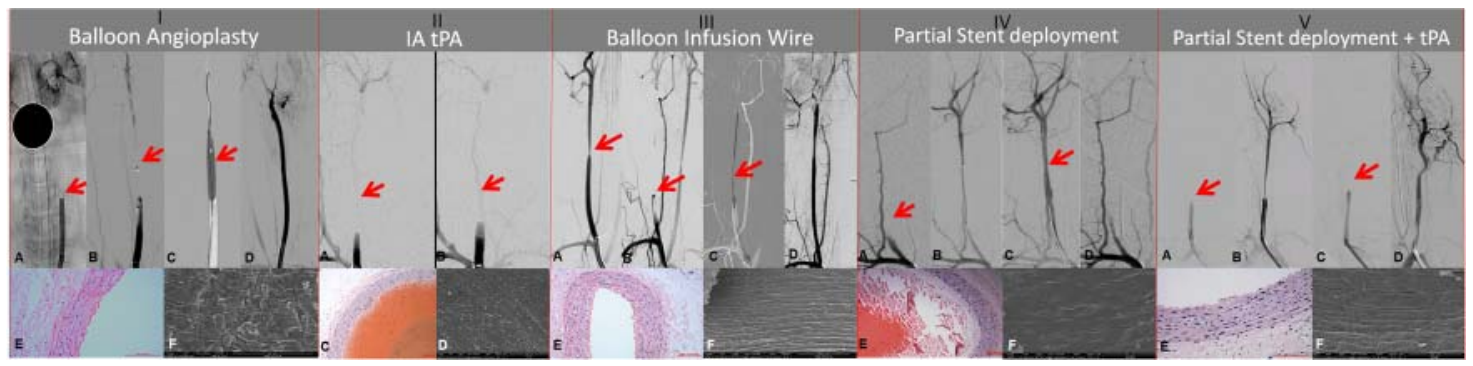

Figure 3 Representative results of device efficacy and safety evaluation. (I) Balloon angioplasty: a stable occlusion is confirmed on digital subtraction angiography (DSA) post clot injection and suture removal (arrow, A). A compliant balloon is positioned at the occlusion site (arrow, B) and balloon angioplasty is performed (arrow, C). Post treatment a final revascularization score of Thrombolysis in Cerebral Infarction (TICI) $2 \mathrm{~A}$ is achieved (D). (II) Intra-arterial tissue plasminogen activator (tPA) when administered at the occlusion site (A, arrow) at a dose of $2 \mathrm{mg}$ fails to revascularize the vessel $(\mathrm{B}, \mathrm{TICl} 0)$. (III) Balloon infusion wire: a stenosis is created in the right common carotid artery (arrow, A). Post clot injection and suture removal reveals a stable occlusion (arrow, B). Post angioplasty and infusion of tPA through the balloon infusion wire (arrow, C) and a final revascularization score of TICI 2B is achieved (D). (IV, V) Partial stent deployment without/with tPA reveals post clot injection and suture removal, a stable occlusion (arrow, IV A,V A). Microcatheter is placed beyond the clot (IV B, V B) and the stent is deployed. There is transient restoration of flow with partial stent deployment alone (IV C), however on resheathing the vessel reoccluded (IV D). In (V), the vessel initially occluded after resheathing the stent (V C). However, secondary to the intra-arterial administration of $4 \mathrm{mg} \mathrm{tPA}$, the clot lysed and a final revascularization score of TICI 2A is achieved (V D). Histology and scanning electron microscopy (I, III, IV, V: E and F; II: C and D), respectively, reveal the vascular safety of the treatment. In all cases there is denudation of the endothelium and exposure of an intact internal elastic lamina. (II C) and (IV E) reveal an intra-luminal thrombus.

occluded vessel for the duration the stent was deployed, as previously reported. ${ }^{20}$ However, shortly following resheathing of the device, the vessel reoccluded, probably due to local platelet aggregation. Also, partially unconstraining the stent, with site directed delivery of tPA, produced inferior recanalization of a median TICI 2A. Perhaps the limited recanalization resulted from tPA being carried distally due to the temporary vascular bypass providing modest systemic thrombolysis. Indeed, we observed that a dose of $4 \mathrm{mg}$ delivered intra-arterially may be required to execute clot lysis in this model.



Figure 4 Histological cross section of a vessel treated with balloon angioplasty alone (hematoxylin-eosin stain). Along the luminal aspect of the media, mild medial acellularity is noted.
As with all preclinical models, this model system also has some limitations. The device evaluation is performed in a straight segment in the extracranial circulation. This does not replicate the environment experienced by these devices in the cerebral circulation where the vessels are more tortuous and histologically distinct, lacking an external elastic lamina, scant adventitia and also susceptible to significant vessel straightening as they are untethered to bone, muscle or fascia. Moreover, a larger number of experiments would need to be conducted to conclude the superiority of one treatment modality. Further, this model only represents a large vessel occlusion and does not lead to ischemic stroke where infarct volume and functional outcomes would be valuable metrics for the benefit of the treatment.

In conclusion, we have described a reliable in vivo model system to allow for a controlled evaluation of the efficacy and vascular safety of combined mechanical and pharmacological therapies.

Acknowledgements The authors are grateful to the Neurolnterventional Therapeutics team, Dr Ender Finol, Dr Michele Migliuolo, Mr Yi Yang and Mr Scott Jahrmarkt, for providing the infusion balloon and insightful collaboration.

Contributors MJG: model development, experimental design, data analysis and manuscript writing. RGN: interventional treatment, experimental design, data analysis and manuscript editing. MM: histopathology, data analysis and manuscript writing. JC: model development, data analysis and manuscript editing. AKW: model development, interventional treatment, data analysis and manuscript editing.

Funding This work was funded in part by a research grant from Neurointerventional Therapeutics, Pittsburgh, PA. Support for imaging from Philips Healthcare is gratefully acknowledged. We also acknowledge technology support from the National Center for Research Resources Award Number S10RR027897. The content is solely the responsibility of the authors and does not necessarily represent the official views of the sponsoring agencies.

Competing interests MJG has been a consultant per hour for Micrus Endovascular, Codman Neurovascular and Soteira Inc; receives research support from the National Institutes of Health, Codman Neurovascular, Concentric Medical, Neurointerventional Therapeutics, Sanofi-Aventis, Stryker Neurovascular and Thrombolytic Science Inc. RGN has been on Scientific Advisory Boards for Stryker/Concentric Medical Inc, Covidien/ ev3 Neurovascular Inc, Penumbra, CoAxia Inc, Rapid Medical, Inc, Reverse Medical and Neurointerventional Therapeutics Inc. AKW: board membership for Surpass Medical; consultancy for Johnson and Johnson, Codman Neurovascular and Stryker Neurovascular; grants/grants pending for National Institutes of Health and Philips Healthcare; payment for lectures (including service on Speakers Bureaus) for Harvard 
Medical School and Baptist Healthcare Miami; stock/stock options for Surpass Medical; travel/accommodation/meeting expenses unrelated to activities listed from Surpass Medical, Codman Neurovascular, Stryker Neurovascular, Covidien and ev3.

Ethics approval The study was approved by the university Institutional Animal Care and Use Committee.

Provenance and peer review Not commissioned; externally peer reviewed.

Open Access This is an Open Access article distributed in accordance with the Creative Commons Attribution Non Commercial (CC BY-NC 3.0) license, which permits others to distribute, remix, adapt, build upon this work non-commercially, and license their derivative works on different terms, provided the original work is properly cited and the use is non-commercial. See: http://creativecommons.org/licenses/by-nc/3.0/

\section{REFERENCES}

1. The National Institute of Neurological Disorders and Stroke t-PA Stroke Study Group. Tissue plasminogen activator for acute ischemic stroke. The National Institute of Neurological Disorders and Stroke rt-PA Stroke Study Group. N Engl J Med 1995;333:1581-7.

2. Alexandrov AV. Current and future recanalization strategies for acute ischemic stroke. J Intern Med 2010;267:209-19.

3. Adeoye $\mathbf{0}$, Hornung R, Khatri $\mathrm{P}$, et al. Recombinant tissue-type plasminogen activator use for ischemic stroke in the United States: a doubling of treatment rates over the course of 5 years. Stroke 2011;42:1952-5.

4. del Zoppo GJ, Higashida RT, Furlan AJ, et al. PROACT: a phase II randomized trial of recombinant pro-urokinase by direct arterial delivery in acute middle cerebral artery stroke. PROACT investigators. Prolyse in acute cerebral thromboembolism. Stroke 1998;29:4-11.

5. Gupta R, Vora NA, Horowitz MB, et al. Multimodal reperfusion therapy for acute ischemic stroke: factors predicting vessel recanalization. Stroke 2006;37:986-90.

6. Choi $\mathbf{J H}$, Bateman BT, Mangla S, et al. Endovascular recanalization therapy in acute ischemic stroke. Stroke 2006;37:419-24.

7. Janjua $\mathbf{N}$, Brisman JL. Endovascular treatment of acute ischaemic stroke. Lancet Neurol 2007:6:1086-93

8. Zivin JA, Fisher M, DeGirolami U, et al. Tissue plasminogen activator reduces neurological damage after cerebral embolism. Science 1985;230:1289-92.
9. Higashida RT, Furlan AJ, Roberts $\mathrm{H}$, et al. Trial design and reporting standards for intra-arterial cerebral thrombolysis for acute ischemic stroke. Stroke 2003;34: e109-37.

10. Jamal A, Bendeck M, Langille BL. Structural changes and recovery of function after arterial injury. Arterioscler Thromb 1992:12:307-17.

11. Gralla J, Schroth G, Remonda L, et al. A dedicated animal model for mechanical thrombectomy in acute stroke. AJNR Am J Neuroradiol 2006;27:1357-61.

12. Mruk JS, Zoldhelyi P, Webster MW, et al. Does antithrombotic therapy influence residual thrombus after thrombolysis of platelet-rich thrombus? Effects of recombinant hirudin, heparin, or aspirin. Circulation 1996;93:792-9.

13. Yakovlev SA, Rublenko MV, Izdepsky VI, et al. Activating effect of the plasminogen activators on plasminogens of different mammalia species. Thromb Res 1995; 79:423-8.

14. Korninger C, Collen D. Studies on the specific fibrinolytic effect of human extrinsic (tissue-type) plasminogen activator in human blood and in various animal species in vitro. Thromb Haemost 1981;46:561-5.

15. Chueh JY, Wakhloo AK, Hendricks GH, et al. Mechanical characterization of thromboemboli in acute ischemic stroke and laboratory embolus analogs. AJNR Am J Neuroradiol 2011;32:1237-44.

16. Siller-Matula JM, Plasenzotti R, Spiel A, et al. Interspecies differences in coagulation profile. Thromb Haemost 2008; 100:397-404.

17. Pasternak RC, Baughman KL, Fallon JT, et al. Scanning electron microscopy after coronary transluminal angioplasty of normal canine coronary arteries. Am J Cardiol 1980:45:591-8

18. Block PC, Fallon JT, Elmer D. Experimental angioplasty: lessons from the laboratory. AJR Am J Roentgenol 1980;135:907-12.

19. US Department of Health and Human Services, Food and Drug Administration, Center for Devices and Radiological Health. Guidance for industry and FDA staff: pre-clinical and clinical studies for Neurothrombectomy devices. Document issued on June 18, 2007. http://www.fda.gov/MedicalDevices/ DeviceRegulationandGuidance?/GuidanceDocuments/ucm071403.htm (accessed 26 Aug 2012)

20. Kelly ME, Furlan AJ, Fiorella D. Recanalization of an acute middle cerebral artery occlusion using a self-expanding, reconstrainable, intracranial microstent as a temporary endovascular bypass. Stroke 2008:39:1770-3. 